\title{
Prognostic significance of the postoperative/ preoperative serum CEA level ratio in patients with solitary adrenal metastasis from lung cancer
}

\author{
SHUGO YAJIMA $^{1}$, YASUKAZU NAKANISHI ${ }^{1}$, SHUNYA MATSUMOTO ${ }^{1}$, KENJI TANABE ${ }^{1}$, \\ MASAHIRO TSUBOI $^{2}$ and HITOSHI MASUDA ${ }^{1}$
}

Departments of ${ }^{1}$ Urology and ${ }^{2}$ Pulmonology, National Cancer Center Hospital East, Kashiwa, Chiba 277-8577, Japan

Received April 27, 2021; Accepted October 6, 2021

DOI: $10.3892 / \mathrm{mco} .2021 .2443$

\begin{abstract}
The present study aimed to evaluate the prognostic significance of the postoperative to preoperative serum carcinoembryonic antigen (CEA) level ratio (CEA ratio) in patients with lung cancer with solitary metastasis of the adrenal gland after adrenalectomy. Furthermore, the optimal CEA ratio cutoff value for predicting disease-free survival (DFS) in patients with lung cancer after adrenalectomy was determined. The medical records of patients with lung cancer with isolated adrenal metastasis who underwent adrenalectomy at the National Cancer Center Hospital East (Kashiwa, Japan) between January 2013 and December 2020 were retrospectively reviewed. Receiver operating characteristic curve analysis was used to segregate patients into two groups (high and low CEA groups) and the clinical prognosis for the two groups was determined. In addition, the association between the CEA ratio and clinical factors was determined. A total of 14 patients with a median age of 68 years (range, 50-75 years) were analyzed, of which $9(64 \%)$ were males. The optimal cutoff value for the CEA ratio to predict DFS was 0.60. The high CEA ratio group $(\geq 0.60)$ displayed poorer DFS and cancer-specific survival ( $\mathrm{P}=0.03$ and 0.02 , respectively). The CEA ratio was significantly associated with the preoperative CEA level $(\mathrm{P}=0.01)$ and the high CEA ratio $(\geq 0.60)$ group had lower preoperative CEA levels. The study suggested that
\end{abstract}

Correspondence to: Dr Yasukazu Nakanishi, Department of Urology, National Cancer Center Hospital East, 6-5-1 Kashiwa no ha, Kashiwa, Chiba 277-8577, Japan

E-mail: yanakani@east.ncc.go.jp

Abbreviations: ASA-PS, American Society of AnesthesiologistsPhysical Status; CEA, carcinoembryonic antigen; CEA ratio, postoperative blood serum CEA level/preoperative blood serum CEA level; CSS, cancer-specific survival; cN stage, clinical node stage; cT stage, clinical tumor stage; DFS, disease-free survival; EBL, estimated blood loss; IQR, interquartile range; ROC, receiver operating characteristic

Key words: adrenal gland, adrenal metastasis, CEA, lung cancer the perioperative CEA ratio may be an important emerging prognostic factor for patients with lung cancer with solitary adrenal gland metastasis. As the sample size was limited, a further study with a larger cohort is required to validate the present findings.

\section{Introduction}

Lung cancer has a high mortality rate for both males and females (1). At the time of diagnosis, $\sim 50 \%$ of the patients with lung cancer have reached a state of distant metastases (2). Approximately $30 \%$ of the metastatic lung cancers are determined to be oligometastatic $(3,4)$. The overall survival for oligometastatic lung cancer was reported to exceed the generally expected 5-year survival of the widespread metastatic disease (30 vs. $4-6 \%)(5,6)$.

In patients with non-small cell lung cancer, isolated metastasis to the adrenal glands, a retroperitoneal organ, occurs in up to $20 \%$ of patients with metastases, among potential sites of dissemination $(4,7)$. However, there is a paucity of such reports in the literature and most of them are small case series $(5,6,8)$. Patients with isolated synchronous or metachronous adrenal metastasis may be offered surgery as part of multimodality treatment for lung cancer. In general, urologists are in charge of the surgical treatment of metastatic adrenal tumors.

Carcinoembryonic antigen (CEA) is one of the most commonly used prognostic markers for lung cancer. Recent studies in the field of colorectal cancer suggested that the post/preoperative serum CEA ratio is able to predict the prognosis of cancer patients $(9,10)$. A retrospective study of 207 patients who underwent resection for colorectal cancer in a single center indicated that the post/preoperative serum CEA ratio was beneficial in predicting prognosis. The overall survival rates were 65.1 and $86.3 \%$ in patients with high $(\geq 0.5)$ vs. low $(<0.5)$ post/preoperative CEA ratios, respectively ( $\mathrm{P}=0.045$, log-rank test) $(10)$. The relevance of the post/preoperative serum CEA ratio has been investigated in the field of primary treatment of lung cancer $(11,12)$, but it has not been previously investigated in the field of lung cancer with solitary metastasis to the adrenal gland, to the best of our knowledge. In the present study, it was hypothesized that the post/preoperative serum CEA ratio is beneficial in predicting 
the postoperative prognosis of solitary adrenal metastasis in lung cancer.

Thus, the present study aimed to investigate the prognostic value of the post/preoperative serum CEA ratio in patients with lung cancer with adrenal metastasis who underwent surgery on the adrenal gland.

\section{Materials and methods}

Study population. The medical records of patients with lung cancer along with synchronous (metastases existing at diagnosis of primary cancer) or metachronous isolated adrenal metastasis who underwent adrenalectomy at the National Cancer Center Hospital East (Kashiwa, Japan) during the period from January 2013 to December 2020, were retrospectively reviewed.

The retrospective study was ethically approved by the National Cancer Center Institutional Review Board (approval no. 2018-159).

Indications for adrenalectomy. At our institute, the indications for adrenalectomy in lung cancer with adrenal metastasis were based on the following criteria: i) The primary lung cancer had been resected or expected to be cured by radical chemotherapy; ii) metastasis was restricted to the adrenal gland; iii) the patient was sufficiently physically fit to tolerate the double-site (lung and adrenal gland) local treatments; iv) the operative risk was considered low based on respiratory reserve and cardiac assessment; v) the patient clearly expressed volition to adhere to heavy multidisciplinary treatment. With the above criteria for adrenalectomy, the final decision was to be made through discussion between the urologist and the pulmonologist.

Collection of serum CEA levels and baseline clinicopathological characteristics. Circulating levels of serum CEA were analyzed with an electrochemiluminescence immunoassay using the Cobas 8000 e602 modular analyzer series (Hoffmann-La Roche AG) as part of the routine care for patients both preoperatively and postoperatively. With the date of measurement determined by the pulmonologist in charge, the CEA ratio as postoperative serum CEA level/preoperative serum CEA level was calculated.

The clinicopathological characteristics, such as age, sex, Charlson co-morbidity index, location of the tumor, metastasis, timing of metastases, tumor staging, treatment of the primary tumor, surgical procedure, perioperative data of adrenalectomy [including operative time, estimated blood loss (EBL) and postoperative complications, which were scored according to the Clavien-Dindo classification (13)], were recorded.

Survival follow-up. The routine follow-up examinations included blood tests such as serum tumor biomarkers such as CEA, as well as imaging diagnostics, including X-rays, positron emission tomography, magnetic resonance imaging and computed tomography. Based on these results, the presence or absence of cancer recurrence was determined.

Disease-free survival (DFS) was calculated from the date of adrenalectomy to the date of recurrence, metastasis or death from any cause. Cancer-specific survival (CSS) was calculated from the date of adrenalectomy to the date of death from lung cancer.
Statistical analysis. The time-dependent receiver operating characteristic (ROC) curve analysis and the Youden index were used to determine the optimal cutoff value for the CEA ratio to predict DFS (14). This was followed by stratification of the eligible patients into the high CEA ratio group and low CEA ratio group.

The survival curve was estimated by the Kaplan-Meier method and statistically significant differences between the groups were determined using a log-rank test.

Mean values with standard deviations were used to express continuous variables with a normal distribution and median values with interquartile ranges (IQR) were used for those with a non-normal distribution. An unpaired t-test or Mann-Whitney U test (continuous variables) and Fisher's exact test (categorical variables) were used to assess the association between the CEA ratio and clinical factors.

All P-values were two-sided and $\mathrm{P}<0.05$ was considered to indicate a statistically significant difference. The statistical analyses utilized in the present study were performed using Microsoft Excel 2016 (Microsoft Corporation) and JMP 13 software (SAS Institute Inc.).

\section{Results}

Patient inclusion. During the study period, 20 adrenalectomies for adrenal metastasis were performed at our institute. A total of 5 (25\%) patients were excluded from the analysis due to having metastases of cancer other than lung cancer; furthermore, $1(5 \%)$ patient was excluded as the intraoperative findings suggested cancer invasion to the head of the pancreas, which resulted in cancelation of the surgery.

Thus, a total of 14 patients with a median age of 68 years (range, 50-75 years), of which 9 (64\%) were males, were included in the study (Table I).

Optimal cutoff value for the CEA ratio to predict DFS. The median preoperative serum CEA level was 10.3 (IQR, 4.8-15.1) $\mathrm{ng} / \mathrm{ml}$ and the measurement date of CEA was a median of 13 (IQR, 3-48) days prior to the adrenalectomy. The median postoperative serum CEA level was 4.6 (IQR, 2.4-6.6) ng/ml and the measurement date of CEA was a median of 33 (IQR, 22-63) days after the adrenalectomy (data not shown).

The median CEA ratio was 0.46 (IQR, 0.32-0.73) ng/ml (data on individual CEA levels for all included patients are not shown). To determine the optimal CEA ratio cutoff value for predicting DFS, a time-dependent ROC curve analysis and the Youden index were used. The optimal cutoff point for the CEA ratio was 0.60 with $60 \%$ sensitivity and $100 \%$ specificity (data not shown). Thus, the patients were categorized into a high CEA ratio $(\geq 0.60)$ group and low CEA ratio $(<0.60)$ group (data not shown).

Clinicopathological patient characteristics. During the follow-up of the 14 eligible patients, 10 (71\%) patients had experienced recurrence of lung cancer with a median time to recurrence of 4.1 months (range, 2.1-43.9 months). Cancer recurrence was identified based on CEA, as well as image diagnostics including X-rays, positron emission tomography, magnetic resonance imaging and computed tomography (data not shown). 
Table I. Comparison of baseline demographic, clinical and pathological characteristics according to CEA ratio.

\begin{tabular}{|c|c|c|c|c|}
\hline \multirow[b]{2}{*}{ Variable } & \multirow[b]{2}{*}{ Total $(n=14)$} & \multicolumn{2}{|c|}{$\mathrm{CEA} \mathrm{ratio}^{\mathrm{a}}$} & \multirow[b]{2}{*}{ P-value } \\
\hline & & $\operatorname{High}(n=6)$ & Low $(n=8)$ & \\
\hline Age, years & $68(60,69)$ & $68(62,71)$ & $67(56,70)$ & 0.46 \\
\hline Sex & & & & 0.33 \\
\hline Male & $9(64)$ & $3(50)$ & $6(75)$ & \\
\hline Female & $5(36)$ & $3(50)$ & $2(25)$ & \\
\hline Charlson co-morbidity index & $0(0,0)$ & $0(0,1)$ & $0(0,0)$ & 0.17 \\
\hline Primary lung cancer treatment & & & & 0.33 \\
\hline Surgery & $9(64)$ & $3(50)$ & $6(75)$ & \\
\hline Other & $5(36)$ & $3(50)$ & $2(25)$ & \\
\hline Histologic type of lung cancer & & & & 0.83 \\
\hline Adenocarcinoma & $12(86)$ & $5(83)$ & $7(88)$ & \\
\hline Small cell & $2(14)$ & $1(17)$ & $1(13)$ & \\
\hline Metachronous & $10(71)$ & $4(67)$ & $6(75)$ & 0.73 \\
\hline cT stage & & & & 0.07 \\
\hline $\mathrm{T} 1$ & $7(50)$ & $4(67)$ & $3(38)$ & \\
\hline $\mathrm{T} 2$ & $3(21)$ & $0(0)$ & $3(38)$ & \\
\hline $\mathrm{T} 3$ & $2(14)$ & $2(33)$ & $0(0)$ & \\
\hline $\mathrm{T} 4$ & $2(14)$ & $0(0)$ & $2(25)$ & \\
\hline cN stage & & & & 0.25 \\
\hline N0 & $7(50)$ & $2(33)$ & $5(63)$ & \\
\hline N1 & $4(29)$ & $2(33)$ & $2(25)$ & \\
\hline $\mathrm{N} 2$ & $1(7)$ & $0(0)$ & $1(13)$ & \\
\hline N3 & $2(14)$ & $2(33)$ & $0(0)$ & \\
\hline Stage & & & & 0.30 \\
\hline I (IA, IB) & $3(21)$ & $0(0)$ & $3(38)$ & \\
\hline II (IIA, IIB) & $3(21)$ & $1(17)$ & $2(25)$ & \\
\hline III (IIIA, IIIB) & $3(21)$ & $2(33)$ & $1(13)$ & \\
\hline IV & $5(36)$ & $3(50)$ & $2(25)$ & \\
\hline
\end{tabular}

${ }^{a}$ The optimal cutoff point for the CEA ratio to predict disease-free survival was 0.60 ; patients were divided into the high CEA ratio ( $\geq 0.60$ ) group and low CEA ratio $(<0.60)$ group. Values are expressed as the median (interquartile range) or $\mathrm{n}(\%)$. CEA, carcinoembryonic antigen; CEA ratio, postoperative blood serum CEA level/preoperative blood serum CEA level; cT stage, clinical tumor stage; cN stage, clinical node stage.

A total of $5(36 \%)$ patients died of lung cancer and the median time from adrenalectomy to death was 29.0 months (range, 6.9-36.1 months). Furthermore, 1 (7\%) patient died 24.9 months after adrenalectomy from a cause other than lung cancer. The five-year survival rate for the eligible patients in the present study stood at $41.0 \%$ with a median survival time of 31.0 months.

Demographic, clinical and pathological characteristics of the patients are presented in Table I. Out of the 14 patients included in the study, 10 patients $(71 \%)$ were observed to have metachronous disease and 4 (29\%) had synchronous metastatic disease. In 9 patients $(64 \%)$, the primary tumor was treated with surgery, 4 patients $(29 \%)$ were treated by thoracic chemo-radiotherapy and 1 patient (7\%) was treated by chemotherapy alone. No significant differences were observed between patients in the high CEA ratio group and low CEA ratio group for the above parameters, age, sex, Charlson co-morbidity Index and histological type of lung cancer.
Similarly, there were no significant differences in the distribution of clinical tumor stage and clinical node stage between the high CEA ratio group and low CEA ratio group.

Peri-operative parameters of adrenalectomy. Peri-operative parameters are presented in Table II. The median operating time for adrenalectomy was $145 \mathrm{~min}$ (IQR, 100-175 $\mathrm{min}$ ), while the median EBL was $19 \mathrm{ml}$ (IQR, 4-56 ml).

Of the 14 patients, 4 patients $(29 \%)$ underwent open surgery ( 3 by retroperitoneal approach) and 10 patients ( $71 \%$ ) underwent laparoscopic adrenalectomy (3 by retroperitoneal approach). Among the 10 patients who underwent laparoscopic adrenalectomy, 1 patient (10\%) required open conversion due to bleeding from the inferior vena cava.

There were no cases of postoperative complications, except for 1 case (7\%) of Clavien-Dindo grade 2 complications (post-operative blood transfusion). 
Table II. Comparison of peri-operative parameters according to CEA ratio.

\begin{tabular}{|c|c|c|c|c|}
\hline \multirow[b]{2}{*}{ Variable } & \multirow[b]{2}{*}{ Total $(n=14)$} & \multicolumn{2}{|c|}{ CEA ratio ${ }^{a}$} & \multirow[b]{2}{*}{ P-value } \\
\hline & & $\operatorname{High}(\mathrm{n}=6)$ & Low $(n=8)$ & \\
\hline Tumor location & & & & 0.12 \\
\hline Left & $8(57)$ & $4(67)$ & $2(25)$ & \\
\hline Right & $6(43)$ & $2(33)$ & $6(75)$ & \\
\hline ASA-PS & $2(1,2)$ & $2(2,2)$ & $2(1,2)$ & 0.21 \\
\hline Preoperative CEA, ng/ml & $10.3(4.8,15.1)$ & $4.5(2.2,9.8)$ & $13.4(10.0,17.8)$ & 0.01 \\
\hline Postoperative CEA, ng/ml & $4.6(2.4,6.6)$ & $4.9(1.9,9.6)$ & $4.5(2.7,6.2)$ & 0.51 \\
\hline Operative time, minutes & $145(100,175)$ & $163(113,190)$ & $130(97,168)$ & 0.80 \\
\hline $\mathrm{EBL}, \mathrm{ml}$ & $19(4,56)$ & $35(10,117)$ & $12(1,59)$ & 0.51 \\
\hline Type of surgical procedure & & & & 0.23 \\
\hline Transabdominal & $1(7)$ & $1(17)$ & $0(0)$ & \\
\hline Retroperitoneal & $3(21)$ & $2(33)$ & $1(13)$ & \\
\hline Transabdominal (laparoscopic) & $7(50)$ & $3(50)$ & $4(50)$ & \\
\hline Retroperitoneal (laparoscopic) & $3(21)$ & $0(0)$ & $3(38)$ & \\
\hline $\begin{array}{l}\text { Post-operative complications, } \\
\text { highest Clavien-Dindo grade }\end{array}$ & & & & 0.37 \\
\hline 0 & $13(93)$ & $6(100)$ & $7(88)$ & \\
\hline I & $0(0)$ & $0(0)$ & $0(0)$ & \\
\hline II & $1(7)$ & $0(0)$ & $1(13)$ & \\
\hline$\geq \mathrm{III}$ & $0(0)$ & $0(0)$ & $0(0)$ & \\
\hline
\end{tabular}

${ }^{a}$ The optimal cutoff point for the CEA ratio to predict disease-free survival was 0.60 ; patients were divided into the high CEA ratio ( $\left.\geq 0.60\right)$ group and low CEA ratio $(<0.60)$ group. Values are expressed as the median (interquartile range) or n $(\%)$. ASA-PS, American Society of Anesthesiologists-Physical Status; CEA, carcinoembryonic antigen; CEA ratio, postoperative blood serum CEA level/preoperative blood serum CEA level; EBL, estimated blood loss.

Preoperative CEA levels were observed to be significantly higher in the low CEA ratio group as compared to the high CEA ratio group $(\mathrm{P}=0.01)$.

Prognostic significance of CEA ratio. As presented in Fig. 1, patients with a high CEA ratio had significantly poorer DFS $(\mathrm{P}=0.03$; Fig. 1A) and CSS $(\mathrm{P}=0.02$; Fig. 1B) as compared to patients with a low CEA ratio. Similarly, for OS, patients with a high CEA ratio tended to have poorer OS than those with a low CEA ratio; however, the difference was not statistically significant ( $\mathrm{P}=0.10$; Fig. $1 \mathrm{C})$.

\section{Discussion}

The prognosis of lung cancer associated with adrenal metastasis is generally poor. However, it has been reported that surgical resection of adrenal metastasis may increase survival when metastasis of the disease is limited to the adrenal gland $(2,5,6,8,15)$.

In the present study, adrenalectomy was performed for lung cancer with solitary metastasis to the adrenal gland. A 5-year survival rate of $41.0 \%$ and a median survival of 31.0 months were determined. This was a relatively high survival rate for metastatic lung cancers. Similar results have been obtained by previous studies of lung cancers with adrenal solitary metastasis, wherein patients underwent adrenalectomy $(6,16)$.
The serum CEA level has been commonly used to evaluate the prognosis of patients with lung cancer. However, serum CEA is not specific to the diagnosis of lung cancer, as CEA levels have been reported to be frequently elevated in patients with colorectal cancer, long-term smoking habit, cardiovascular disease, gynecological disease and other diseases (9). In the case of colorectal cancer, it has been reported that clinical prognosis may be predicted by evaluating not only the preoperative CEA level but also the post/preoperative serum CEA ratio $(9,10)$. A retrospective single-center study including 187 patients with colorectal cancer suggested that the CEA ratio was not able to improve the predictive efficiency for prognosis as compared to postoperative CEA levels in terms of sensitivity and specificity. Furthermore, the CEA ratio was indicated to be useful as a prognostic indicator for patients with colorectal cancer and normal postoperative CEA by Kaplan-Meier survival curve and subgroup multivariate analysis (9).

The present study indicated for the first time, to the best of our knowledge, DFS of patients with a high CEA ratio $(\geq 0.60)$ was significantly poorer compared to those with a low CEA ratio $(<0.60)$ after adrenalectomy for solitary adrenal metastasis from lung cancer. In addition, the CSS was determined to be significantly poorer in the group with a high CEA ratio $(\geq 0.60)$. There was no significant difference in OS, although 

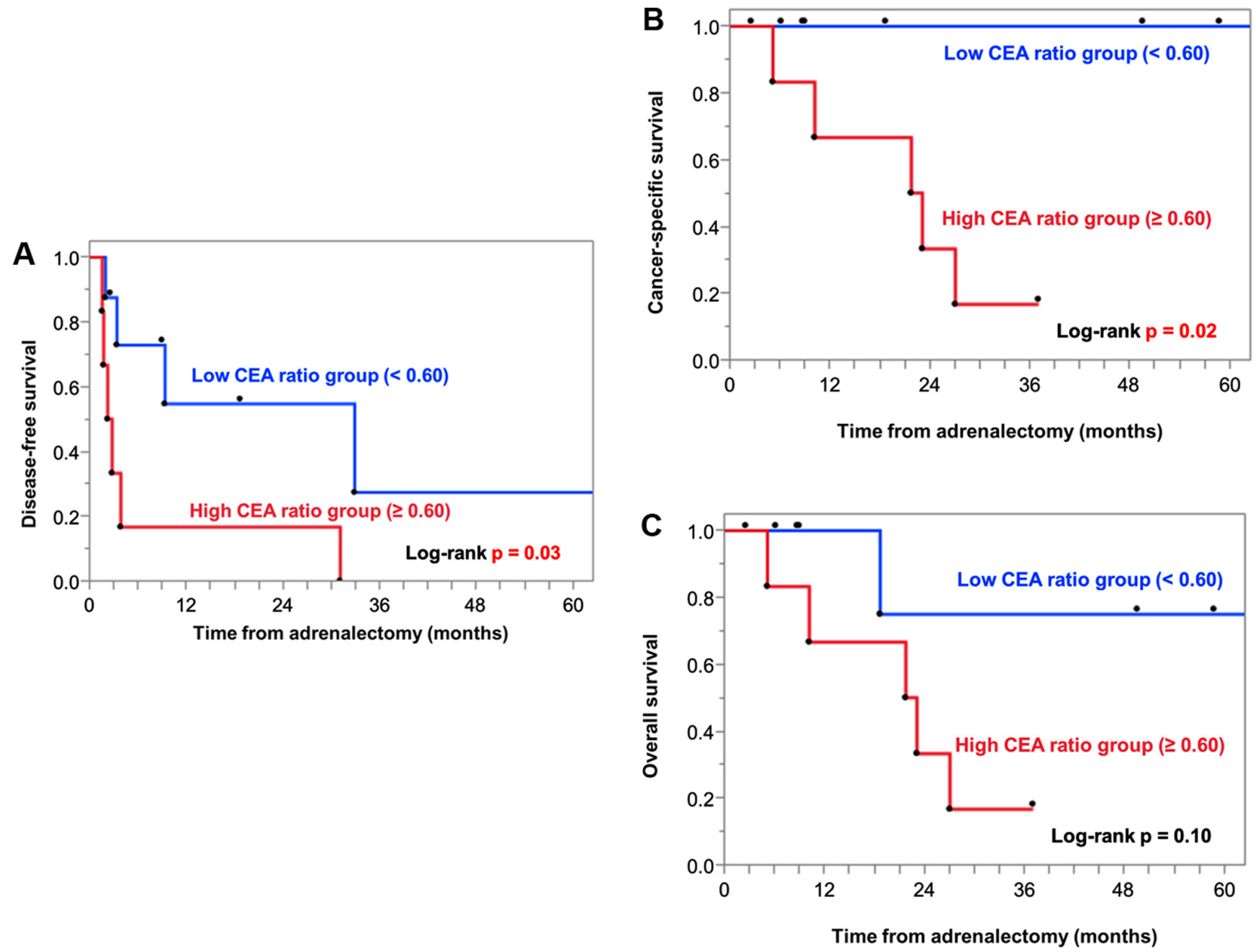

Figure 1. Comparisons of Kaplan-Meier curves of (A) DFS, (B) CSS and (C) overall survival between two groups (high CEA ratio, $\geq 0.60$; low CEA ratio, $<0.60$ ). When 0.60 was set as the cutoff for the CEA ratio, patients with a high CEA ratio had significantly poorer DFS and CSS than those with a low CEA ratio. CEA, carcinoembryonic antigen; CEA ratio, post/preoperative serum CEA levels; DFS, disease-free survival; CSS, cancer-specific survival.

there was a trend toward unfavorable OS in the group with a higher CEA ratio $(\geq 0.60)$; this may be worth examining to observe what the results would be if the sample size were larger. The group with a lower CEA ratio $(<0.60)$ exhibited higher preoperative CEA levels than their counterpart. On the other hand, there was no significant difference in postoperative CEA levels between the two groups. These results suggested the relevance of not only evaluating preoperative or postoperative CEA values but also evaluating the CEA ratio in predicting prognosis after adrenalectomy for lung cancer with solitary adrenal metastasis. Furthermore, there was no statistically significant difference in the distribution of the clinical $\mathrm{T}$ or $\mathrm{N}$ stage of lung cancer between the high CEA ratio group and the low CEA group. In other words, taking into account the factor of the CEA ratio as well as cancer staging may be a useful adjunct tool in predicting prognosis.

There were no significant differences in postoperative CEA levels between the high CEA ratio and the low CEA ratio groups. Of note, the group with a lower CEA ratio $(<0.60)$ had higher preoperative CEA levels. A similar trend was observed in a previous study that examined the prognostic value of the CEA ratio in colorectal cancer (9). Cases of lung cancer with high preoperative CEA levels may be considered as a high-CEA secretion type and the changes in CEA levels may reflect the degree of residual tumor cells in the body. The fact that no experiments were performed to demonstrate this hypothesis is a limitation of the present study.

The present study had several other limitations. First, this retrospective study was a single cohort study that was based on the limited data available. Owing to the small sample size, no definitive independent variable to predict prognosis was determined. Furthermore, the study did not have any fixed protocol for the date of measurement of the serum CEA levels, and therefore, there was variation in the measurement dates, as measurements were performed at a median of 13 (IQR, 3-48) days prior to surgery and a median of 33 (IQR, 22-63) days after surgery. In general, the half-life of CEA values is 3-7 days (12,17-19) and therefore, the different measurement dates from the postoperative period may have resulted in different CEA levels even in the same patient. This may therefore raise the concern about the reproducibility of the analysis results regarding that the CEA ratio is able to predict patient prognosis. Finally, the interpretation of the present findings is limited by losses to follow-up (two patients were lost to follow-up; the observation periods were 247 days and 2,351 days, respectively). 
In conclusion, the present study suggested that the perioperative CEA ratio may be a simple and important emerging prognostic factor for patients with lung cancer with solitary adrenal gland metastasis. However, this was a retrospective study with a small sample size and validation in a future prospective study with an appropriate sample size is essential for the generalization of this finding and assessment of applicability in clinical practice.

\section{Acknowledgements}

Not applicable.

\section{Funding}

No funding was received.

\section{Availability of data and materials}

The datasets used and/or analyzed during the current study are available from the corresponding author upon reasonable request.

\section{Authors' contributions}

SY, YN and HM conceived the present study. SY, KT and MT designed the study. KT and SM acquired the data. SY, KT and SM analyzed and interpreted the data and drafted the manuscript. YN performed critical revision of the manuscript. MT and HM supervised the study and revised the manuscript critically for important intellectual content. All authors read and approved the final manuscript. YN and HM checked and approved the authenticity of the raw data. All authors agree to be accountable for all aspects of the work in ensuring that questions related to the accuracy or integrity of any part of the work are appropriately investigated and resolved (according to the ICMJE).

\section{Ethics approval and consent to participate}

This study is retrospective and informed consent from the patients was not required. The present study was approved by the National Cancer Center Institutional Review Board (Kashiwa, Japan; approval no. 2018-159).

\section{Patient consent for publication}

Not applicable.

\section{Competing interests}

The authors declare that they have no competing interests.

\section{References}

1. Jemal A, Bray F, Center MM, Ferlay J, Ward E and Forman D Global cancer statistics. CA Cancer J Clin 61: 69-90, 2011.
2. Walters S, Maringe C, Coleman MP, Peake MD, Butler J, Young N, Bergström S, Hanna L, Jakob-sen E, Kölbeck K, et al; ICBP Module 1 Working Group: Lung cancer survival and stage at diagnosis in Australia, Canada, Denmark, Norway, Sweden and the UK: A population-based study, 2004-2007. Thorax 68: 551-564, 2013.

3. Albain KS, Crowley JJ, LeBlanc M and Livingston RB: Survival determinants in extensive-stage non-small-cell lung cancer: The Southwest Oncology Group experience. J Clin Oncol 9: 1618-1626, 1991.

4. Eberhardt WEE, Mitchell A, Crowley J, Kondo H, Kim YT, Turrisi A, Goldstraw P and Rami-Porta R: The IASLC Lung Cancer Staging Project: Proposals for the revision of the M descriptors in the forthcoming eighth edition of the TNM classification of lung cancer. J Thorac Oncol 10: 1515-1522, 2015.

5. Raz DJ, Lanuti M, Gaissert HC, Wright CD, Mathisen DJ and Wain JC: Outcomes of patients with isolated adrenal metastasis from non-small cell lung carcinoma. Ann Thorac Surg 92: 1788-1793, 2011.

6. Gao XL, Zhang KW, Tang MB, Zhang KJ, Fang L-N and Liu W: Pooled analysis for surgical treatment for isolated adrenal metastasis and non-small cell lung cancer. Interact Cardiovasc Thorac Surg 24: 1-7, 2017.

7. Higashiyama M, Doi O, Kodama K, Yokouchi H, Imaoka S and Koyama H: Surgical treatment of adrenal metastasis following pulmonary resection for lung cancer: Comparison of adrenalectomy with palliative therapy. Int Surg 79: 124-129, 1994.

8. Luketich JD and Burt ME: Does resection of adrenal metastases from non-small cell lung cancer improve survival? Ann Thorac Surg 62: 1614-1616, 1996.

9. Xie HL, Gong YZ, Kuang JA, Gao F, Tang SY and Gan JL: The prognostic value of the postop-erative serum CEA levels/preoperative serum CEA levels ratio in colorectal cancer patients with high preoperative serum CEA levels. Cancer Manag Res 11: 7499-7511, 2019.

10. Odeny TA, Farha N, Hildebrandand H, Allen J, Vazquez W, Martinez M, Paluri RK and Kasi A: Association between primary perioperative CEA ratio, tumor site, and overall survival in patients with colorectal cancer. J Clin Med 9: 3848, 2020.

11. Nonaka M, Kataoka D, Yamamoto S, Bito A, Matsuoka J, Kawada T and Takaba T: Pre- and post-operative serum carcinoembryonic antigen in primary lung adenocarcinoma. Ann Thorac Cardiovasc Surg 10: 281-284, 2004.

12. Ito K, Hibi K, Ando H, Hidemura K, Yamazaki T, Akiyama S and Nakao A: Usefulness of analyti-cal CEA doubling time and half-life time for overlooked synchronous metastases in colorectal carci-noma. Jpn J Clin Oncol 32: 54-58, 2002.

13. Clavien PA, Barkun J, de Oliveira ML, Vauthey JN, Dindo D, Schulick RD, de Santibañes E, Pekolj J, Slankamenac K, Bassi C, et al: The Clavien-Dindo classification of surgical complications: Five-year experience. Ann Surg 250: 187-196, 2009.

14. Akobeng AK: Understanding diagnostic tests 3: Receiver operating characteristic curves. Acta Pae-diatr 96: 644-647, 2007.

15. Howell GM, Carty SE, Armstrong MJ, Stang MT, McCoy KL, Bartlett DL and Yip L: Outcome and prognostic factors after adrenalectomy for patients with distant adrenal metastasis. Ann Surg Oncol 20: 3491-3496, 2013.

16. Mazzella A, Loi M, Mansuet-Lupo A, Bobbio A, Blons H, Damotte D and Alifano M: Clinical characteristics, molecular phenotyping, and management of isolated adrenal metastases from lung cancer. Clin Lung Cancer 20: 405-411, 2019.

17. Park YA, Lee KY, Kim NK, Baik SH, Sohn SK and Cho CW: Prognostic effect of perioperative change of serum carcinoembryonic antigen level: A useful tool for detection of systemic recurrence in rectal cancer. Ann Surg Oncol 13: 645-650, 2006.

18. Choi JS and Min JS: Significance of postoperative serum level of carcinoembryonic antigen (CEA) and actual half life of CEA in colorectal cancer patients. Yonsei Med J 38: 1-7, 1997.

19. Yakabe T, Nakafusa Y, Sumi K, Miyoshi A, Kitajima Y, Sato S, Noshiro $\mathrm{H}$ and Miyazaki K: Clini-cal significance of CEA and CA19-9 in postoperative follow-up of colorectal cancer. Ann Surg Oncol 17: 2349-2356, 2010. 